\title{
The COST 271 Action: conclusions and the way ahead
}

\author{
LJILJANA R. CANDER $\left({ }^{1}\right)$ and BRUNO ZOLESI $\left({ }^{2}\right)$ \\ ( $\left.{ }^{1}\right)$ Rutherford Appleton Laboratory, Chilton, Didcot, Oxon, U.K. \\ $\left.{ }^{(}\right)$Istituto Nazionale di Geofisica e Vulcanologia, Roma, Italy
}

\begin{abstract}
A brief summary is given of the major achievements of the COST 271 Action. New challenges have been identified that open the way for a proposal, which is outlined, for a follow-on to the COST 271 Action within the COST Telecommunications, Information Science and Technology framework.
\end{abstract}

\section{INTRODUCTION}

The expanding need for new communications services, especially those involving ionospheric HF communications, satellite communications and navigational systems, imposes increasing demands for the continuous monitoring and better understanding of the propagation effects imposed by the Earth's upper atmospheric that play important roles in determining the characteristics and reliability of radio systems. There is a requirement to develop means to predict regular behaviour and to minimise disturbing effects over a wide range of propagation conditions.

Within this context, the COST 271 Action on «Effects of the upper atmosphere on terrestrial and Earth-space communications» has made a significant impact in a number of areas:

a) Progress has been made in the examination of the need for information on propagation effects on both currently-operational and future-anticipated terrestrial and Earth-space radio systems that can be affected by space-weather phenomena, with emphasis being placed on characterisation of the relevant phenomena and their associated consequences.

b) Specific studies have been undertaken in response to the terms of references of COST 271 Action Work Packages.

c) The results from these investigations have been discussed at three Workshops and published to the considerable benefit of both participating and other organisations.

d) New data have been collected and mathematical methods and computational tools developed.

e) Dedicated studies have made a significant input to the emerging science of the impact of space weather on the terrestrial environment and advanced technology.

f) Results of significance have been made available to the ITU-R Study Group 3 for incorporation into international recommendations. Full details of these achievements have been given in the previous sections of this report and placed on the COST 271 Web site (www.cost271.rl.ac.uk). In addition, the CD containing this Final Report includes the full bibliography, addresses of participants and relevant web links.

The major achievements of the COST 271 Action are summarised below, with new challenges being identified and incorporated into a proposal for a follow-on to the Action that is outlined. 


\section{The MAJOR ACHIEVEMENTS OF COST 271}

- A survey has been carried out of modern radio systems in different frequency bands, within the context of international regulations and the propagation phenomena that can lead to system impairments, together with the space-weather effects that can have significant adverse impacts on propagation conditions that need to be modelled.

- Ionospheric stations over Europe have made a vital contribution in maintaining the high standard of vertical-incidence measurements and providing data on a regular basis. Collection and distribution of the historical, new and prompt ionospheric data has been carried out at both the Rutherford Appleton Laboratory (http://www.wdc.rl.ac.uk/cgi-bin/digisondes/cost_database.pl) and the Space Research Centre (http://www.cbk.waw.pl/rwc). In addition, there has been continuing support of the limited database of past ionospheric observations that was developed under the previous COST Action 251 (http://cost251.ictp.trieste.it/).

- A database of EISCAT observations from 1981 to 1999 have been developed at the University of Grenoble (http://www-eiscat.ujf-grenoble.fr).

- Operational services for nowcasting and forecasting the state of the ionosphere over Europe are now available at the Regional Warning Center in Warsaw (http://www.cbk.waw.pl/rwc) and the Rutherford Appleton Laboratory (http://ionosphere.rcru.rl.ac.uk).

- Modelling techniques have been formulated, leading to experimental and real-time operational services involving new mathematical methods and computational tools for the forecasting and regional mapping of ionospheric characteristics.

- Improvements have been made to existing understanding of the physical mechanisms responsible for possible long-term trends in the Earth's ionospheric and atmospheric parameters that may possibly link such trends with anthropogenic activities.

- Studies have been carried out to advance knowledge of the structure and dynamics of the upper atmosphere and provide tools needed for the investigation of thermospheric-ionospheric interactions under various geophysical conditions.

- Models, and other tools to assess ionospheric effects in non-ionospheric applications of GNSS signals, have been investigated at the University of Graz (http://www.uni-graz.at/igamwww/cost271/) including a) ionospheric influences in the use of GNSS occultation for stratosphere/troposphere applications and b) the effect of higher-order ionospheric propagation errors in advanced ground-based applications, like water vapour retrieval.

- GPS-based regional maps and the TECEDA data bank are now available at the Deutsches Zentrum für Luft und Raumfahrt (http://www.kn.nz.dlr.de).

- Collection has been started of examples of ionospheric extremes and anomalous cases with unusually high or low electron content values, anomalous gradients and variations that could be attributed to the influence of magnetic storms, Travelling Ionospheric Disturbances (TIDs) and other as yet partially unexplained effects.

- Investigation has been made of gravity and planetary waves at mid-latitudes, responsible for some of the residual uncertainty in ionospheric radio-wave propagation predictions, with a view to identifying patterns to improve the accuracy of forecasts for telecommunication purposes.

- A physically-based software simulator has been developed for the HF ionospheric reflection channel and UHF simulators for transionospheric channels, which overcome the limitations of existing empirically-based models.

- Studies have been carried out leading to a better understanding of the behavior of the ionospheric $F 1$-region, sporadic $E(E s)$ and spread- $F$ phenomena at European middle latitudes under geomagnetic storm conditions.

- Measurements and simulations have been made and results of importance to applied radio systems obtained, relating to the propagation of HF radio waves over northern European paths, where ionospheric effects impose large Doppler and delay spreads on the propagating signal. 
- A heterogeneous array has been investigated to improve HF transmission, offering the possibility to transmit images via the ionospheric channel by providing an increase of the data rate of 15 $\mathrm{kHz}$ within a $3 \mathrm{kHz}$ bandwidth (QAM-64) without coding or interleaving.

- Improvements to the GISM model include use of the multiple phase screen technique to give statistical characteristics of transmitted signals; fade duration and other parameters like the probability of signal loss of lock. Maps of scintillation index S4 and standard deviations of the phase fluctuations can be produced.

- Algorithms and software tools have been developed to treat disturbances in Earth-space and satellite-to-satellite communications.

- Studies have been made of plasma effects in the magnetosphere-ionosphere-thermosphere system generated by different natural processes and by human activity.

- Information has been gathered about effects of the vertical and horizontal gradients of the electron density on Earth-space and satellite-to-satellite communication.

- Model simulation studies have been made of extreme propagation effects on GPS-to-geostationary satellite ray paths.

\section{Proposal for a follow-on action}

Since 1991, the COST 238, COST 251 and COST 271 Actions have been highly successful in bringing together most of the available European expertise in the field of ionospheric/plasmaspheric research and the application of such knowledge to the improvement of practical radio systems. The important results stemming from these collaborative projects can be found in reports of the earlier Actions and in the current volume for COST 271. The results obtained from the measurements and modelling within these Actions have aided radio-systems planners and designers in two primary respects. Not only is there better understanding of the upper atmosphere environment over Europe and its effects on terrestrial and Earth-space communications and navigational systems, but also it has been possible to determine with greater accuracy the propagation effects imposed by the atmosphere on received signals and consequent interference levels. In addition, each successive project has raised new questions that have been taken up in the next action. COST 271 is no different in this respect. Indeed, it is inevitable that wide-ranging studies of this magnitude, together with the advance of the technological requirements of new generations of communications systems, will raise different technical issues that cannot be properly addressed or completed within the prescribed timeframe of the Action. However, the COST framework provides a unique European forum for the progression of such matters. In consequence, the Management Committee of the COST 271 Action has formulated a Memorandum of Understanding of a new four-year study to follow, in which such questions can be taken up. While COST 271 was concerned with identification and investigation of the propagation effects imposed by the upper atmosphere, the new proposal (MIERS) now plans to deal directly with the mitigation of such effects on practical radio systems.

\subsection{Mitigation of Ionospheric Effects on Radio Systems (MIERS)}

With the following main objectives:

- To support and enhance the existing European facilities for historical and real-time digital ionospheric data collection and to exchange information needed on methods and algorithms to mitigate the effects of ionospheric perturbations and variations on advanced terrestrial and space-based communication services by creating an effective computing infrastructure.

- To develop an integrated approach to ionospheric modelling, create the mechanism needed to ingest processed data into models, extend and develop suitable mitigation models and define the protocols needed to link models together. 
- To make applicable results available to the ITU-R and to promote the research aspects to funding agencies like ESA, ESF and FP 6/7.

- To strengthen the areas of expertise that already exist by stimulating closer cooperation between scientists and users, focusing the scope of all the previous COST ionospheric related studies to the mitigation of ionospheric effects on specific radio systems, which are in operational use or in the development stage into new systems.

The work will be arranged under three topic headings and will progress within each of these areas throughout the duration of the Action (four years) as follows.

\subsubsection{WG 1 - Ionospheric monitoring and modelling}

WP 1 - Near-Earth space plasma monitoring

- Monitoring near-Earth space by vertical incidence and oblique sounding networks and GNSS techniques (retrospective and real-time).

- Ionospheric data networking.

- TEC: model-assisted techniques and measurements from the global network.

WP 2 - Data ingestion and assimilation in ionospheric models

- Data collection and processing.

- Model adaptation for data ingestion and assimilation.

WP 3 - Near-Earth space plasma modelling and forecasting

- Real-time near-Earth space plasma modelling.

- Modelling and forecasting of ionospheric perturbations.

- Tomographic imaging for model validation.

- Channel modelling by neurofuzzy techniques.

WP 4 - Climate of the upper atmosphere

- Long-term ionospheric trends.

- Gravity and planetary wave effects on propagation and infrasonic waves.

- Ionospheric space weather.

\subsubsection{WG 2 - Advanced terrestrial systems}

WP 1 - Radar and radiolocation

- Ionospheric effects on surface-wave radar.

- Frequency management of ground-wave and sky-wave radars.

- Angle of arrival measurements for sky-wave signals.

WP 2 - HF/MF communications

- Digital radio systems - predictions, methods of estimating reliability, etc.

- Wideband propagation modelling and development of a hardware simulator.

- Increased capacity of HF links through MIMO techniques - experimental measurements and simulation.

- Extension of existing wideband HF simulators to the MF band.

WP 3 - Spectrum management

- Use of GPS to improve HF communications management. 
- Adaptive waveform management.

- Effects of infrasound on radio propagation.

\subsubsection{WG 3 - Space-based systems}

WP 1 - Space plasma effects

- Ionospheric effects on space-based remote sensing systems.

- Gravity wave effects on GNSS.

- Space plasma media and security implications.

WP 2 - Mitigation techniques

- Ionospheric structures, their physical nature and impact on GNSS signals.

- Improved accuracy of GNSS by better ionospheric correction.

- Errors due to ionospheric perturbations.

WP 3 - Scintillation monitoring and modelling

- Scintillation effects, their physical nature and impact on ionospheric radio systems, GNSS signals in particular.

- High-latitude scintillation effects: experimental work and modelling.

- Equatorial scintillation effects: experimental work and modelling.

\section{Conclusions}

The COST 271 Action has been very successful in bringing together in collaborative studies many researchers who collectively represent much of the available European expertise on the effects of the upper atmosphere on radio systems. The significant results and advances in knowledge and understanding stemming from COST 271 have been summarized here. In addition, a proposal has been outlined for new investigations within the COST Telecommunications, Information Science and Technology framework into the mitigation of such propagation effects on practical communications systems. 